\title{
DEVELOPMENT OF AN INTELLIGENT ADAPTER FOR FIELD COMPUTER
}

\author{
Wei Yang, Minzan Li, Hui Liu \\ Key laboratory of Modern Precision Agriculture System Integration Research, Ministry of \\ Education, China Agriculture University, Beijing 100083,China.Email: limz@cau.edu.cn
}

Abstract: A kind of intelligent adapter between CAN bus network and PC104 system was developed. The adapter is made up of an interface of CAN bus network, a CAN receiver connecting with the interface, a CAN controller, a PC104 busslot, a high speed opt coupler, a microprocessor and a parallel interface circuit. PC104 system can just take charge of transmitting and receiving data without performing maintenance of $\mathrm{CAN}$ bus because of using the microprocessor. Thus the system resource can be saved for other tasks. The adapter uses parallel communication mode and has high data transmission rate. The drive $\mathrm{VxD}$ of the adapter and the functional module of the PC104 system were programmed. Debugging shows the adapter works stably and satisfactorily.

Key words: $\quad$ PC/104, CAN bus, Field computer, parallel communication mode

\section{INTRODUCTION}

The Controller Area Network (CAN) is a serial communications protocol, which efficiently supports distributed real-time control with a very high level of security. It has been used from high speed networks to low cost multiplex wiring, such as automotive electronics, engine control units, sensors, antiskid brake systems, etc. PC104 system is a new kind of embedded system. It has two bus hubs, P1 and P2. P1 has 64 pins and P2 has 40 pins so it is called PC104 system. The size is very small just $90 \mathrm{~mm} \times 96 \mathrm{~mm}$, and the drive current is low. Thus it fits for using in commercial installation. 
In a word, field computer based on PC104 system and farmland data collection control system based on CAN Bus have been developed. In order to use the field computer as an independent application unit or an independent CAN node for precision agriculture, it is necessary to build a kind of bridge between CAN Bus and PC104 system. Hence, we decided to develop an intelligent adapter between CAN Bus and PC104 system.

\section{MATERIALS AND METHODS}

The development of the intelligent adapter was based on a developed field computer. The field computer was an embedded mobile computer system fixed on farm machinery. It can be used as a real-time information management tool for precision agriculture, such as field data acquisition, variable rate controlling, and so on.

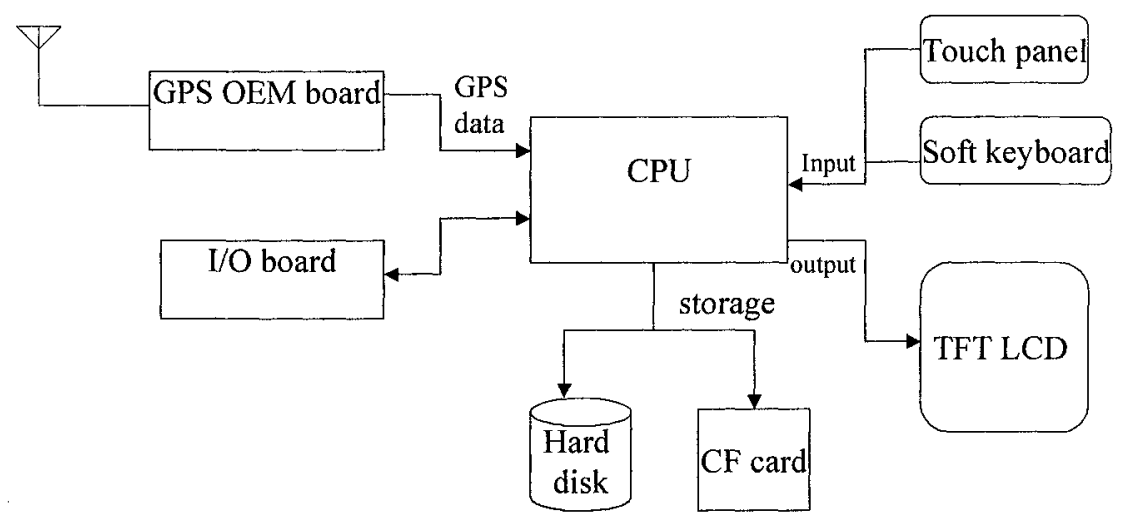

Figure 1. The structure of the field computer

Fig. 1 shows the structure of the field computer. It consisted of a CPU, a GPS OEM board, a I/O board, a touch panel, a soft keyboard, a TFT LCD, a hard disk, and a driver of Compact Flash Card. PCM-3350, a kind of industrial PC/104 motherboard, was chosen as the core of the field computer. A TFT LCD and a tough panel were selected as the output device and the input interface respectively, and a hard disk was taken as storage device. The Field Computer supported RS-232 and USB serial ports. It also supported Compact Flash Card as a mobile storage medium. A GPS-OEM board was embedded, which made the Field Computer have more options on GPS.

The software needed by the computer was programmed. It could meet the field operation demands of precision agriculture. Map Objects 2.0, a kind of 
GIS Active X control from ERST, was chosen as the base of the software to aim at the integration of GPS and GIS in agriculture. The software was developed under the Visual $\mathrm{C}++6.0$ with friendly interface and abundant functions. The software could perform the functions such as GPS data receiving and processing, field mapping, grid sampling, feature logging, parallel swathing, and so on.

\section{DEVELOPMENT OF THE INTELLIGENT ADAPTER}

The adapter based on field computer was made up of an interface of CAN bus network, a CAN receiver connecting with the interface, a CAN controller, a PC/104 bus-slot of the industrial PC/104 motherboard, a high speed opt coupler, a microprocessor, and a parallel interface circuit. Below will introduce the whole design and the working principle of the adapter.

\subsection{Hardware of the adapter:}

The main function of the adapter was to build a bridge between CAN Bus and PC104 system. Thus the adapter was needed to incept data from CAN and then transfer to $\mathrm{PC} 104$ system, and to transfer data from $\mathrm{PC} 104$ system to CAN. In order to meet the demand of the real-time control with a very high level of security in farm data collection, the adapter must have high receiving and transmitting data speed. In order to make the data receiving and data transmitting accurate, the hindrance between PC104 and CAN should be avoided. In the hardware development, a microprocessor was used as the control unit of the adapter, which made the adapter intelligent.

Fig. 2 shows the operational principle of the intelligent adapter. The adapter included: A CAN bus interface used to connect CAN, a CAN BUS receiver $82 \mathrm{C} 250$ used to transmit and receive data from CAN, a CAN controller used to process data and then transmit it to CAN BUS receiver, or to transmit and receive data from CAN BUS receiver, a PC/104 bus-slot used to connect with mainboard, a MCS51 microprocessor (MCU) used to maintain CAN serial, a Parallel interface circuit (8255A) used to transmit data in parallel mode, and a opt coupler used to enhance the anti-jamming of the system. 


\subsection{Design of the Parallel interface circuit}

Since the low speed of the serial communication was not able to match the $\mathrm{CAN}$ bus speed reached $1 \mathrm{MbPs}$, the parallel communication mode was

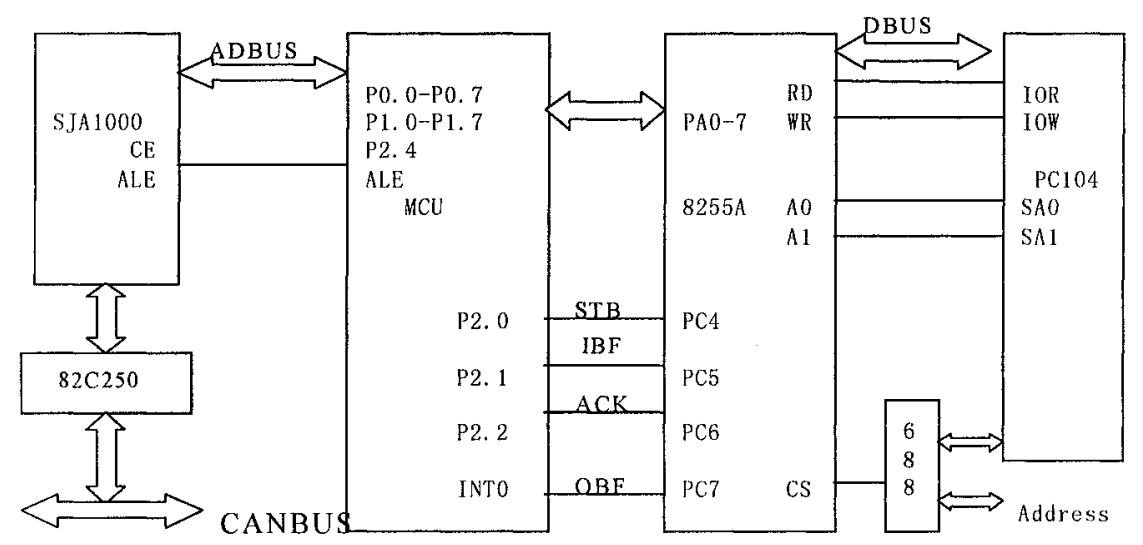

Figure 2.The operational principle of the adapter

used by the adapter. And an $8255 \mathrm{~A}$ was used to directly read and write $\mathrm{I} / \mathrm{O}$ port under the bus timing of PC104.

$8255 \mathrm{~A}$ has three kinds of working modes. Mode 2 was used in data communication, which was bi-directional communication mode and only fit for the port A. Port A was connected with port Plof the MCU to transmit data between $8255 \mathrm{~A}$ and $\mathrm{MCU}$. Port $\mathrm{C}$ of $8255 \mathrm{~A}$ provides control signals to port $A$. The data bus of the $8255 \mathrm{~A}$ was connected with the data bus of the PC104, so that it could transmit data to PC104 serial, and could also receive data from PC104 serial. On addressing selection, PC104 could provide $64 \mathrm{~K}$ $\mathrm{I} / \mathrm{O}$ ports for data communication. We only used four of those to control $8255 \mathrm{~A}$, address $280 \mathrm{H}-283 \mathrm{H}$. When the field computer wanted to transmit data to adapter, $\mathrm{PCl} 104$ serial port sent address signal $282 \mathrm{H}$ firstly, then read port $\mathrm{C}$ to judge if IBF bit was in effect, in aims to judge if there were data in buffer. If yes, delaying for a moment and sending $282 \mathrm{H}$ again. Otherwise sending addresses signal $280 \mathrm{H}$ and then sending data to port $\mathrm{A}$ of $8255 \mathrm{~A}$. At the same time, $8255 \mathrm{~A}$ sent $\mathrm{OBF}$ signal to INT0 of MCU. When MCU received the signal, it would send ACK signal to $8255 \mathrm{~A}$, and $8255 \mathrm{~A}$ would make output buffer in effect and then send data to the buffer.

When data were transmitted to the field computer, MCU received the data from CAN controller and judged if IBF was in effect. If input buffer 
was full, sending data to port A of $8255 \mathrm{~A}$, and at the same time sending STB signal. The computer queried IBF bit of port $C$ to judge if IBF bit was in effect. If yes it would take the data from input buffer of $8255 \mathrm{~A}$.

\subsection{Design of CAN controller interface}

A CAN controller SJA1000 and a CAN receiver $82 \mathrm{C} 250$ were used as the CAN interface of the adapter. In order to enhance the ability of antidisturbance, the adapter adopted two high-speed opt couplers installed between SJA1000 and 82C250 and a DC/DC power supply for the opt couplers. MCU was the key of the whole adapter since it took charge of the communication between $8255 \mathrm{~A}$ and CAN controller.

\subsection{Software of the adapter}

The adapter mainly took charge of data translating between PC104 and CAN node. The designed software included two parts. One was the adapter driver $\mathrm{VxD}$ and the initialization of CAN interface. The other was MCU control program.

\section{a) Adapter driver $\mathrm{VxD}$}

The driver mainly included interface function to take charge of the communication between the field computer and 8255A. The programming of the driver was based on Visual Basic 6.0. The adapter must compile VxD (virtual device driver) to call the low-level hardware of the computer. We used DLL to encapsulate $\mathrm{VxD}$ so that users could use $\mathrm{VxD}$ more conveniently. The DLL provided several functions such as port setting, port closing, port writing, and port reading.

b) Initialization of CAN interface and MCU control program

The MCU control program was used to take charge of the communication between $8255 \mathrm{~A}$ and CAN controller. It was finished by ASM51 assemble language. The main tasks of the software were: translating the commands of the computer to the CAN controller, and then transmitting them to CAN Bus; and transmitting all kinds of state from CAN to $8255 \mathrm{~A}$.One of the key points of the software was the initialization of the CAN controller SJA1000. 


\section{DEBUGGING AND CAPABILITY EVALUATION}

Using a base CAN node the adapter was tested for simulation debugging and capability evaluation. Simulation debugging included two procedures: self-transmitting and self-receiving test and node-to-node test.

a) Self-transmitting and self-receiving test

CAN controller SJA1000 has the function of self-transmitting and selfreceiving data. When setting the special bit of SJA1000, it would be possible to conduct self-transmitting and self-receiving data test. The CAN part of the adapter followed CAN 2.0B protocol, and could work on PELICAN mode. The result showed that the data in output buffer of SJA1000 was the same as the data in input buffer of SJA1000. It proved that the CAN mode could work up to snuff.

\section{b) Node to node test}

Using an independent CAN node to communicate with the adapter, the node-to-node test was executed. Figure 3 shows the interface of the test. 【 input port address】 was the CS of 8255A. 【 querying state port 】 displayed input address of state port. 【output data】 and 【input data】 showed the data received from CAN bus and the data from PC104 system respectively.【input data show】and【output data show】 showed the data has been received or transmitted.

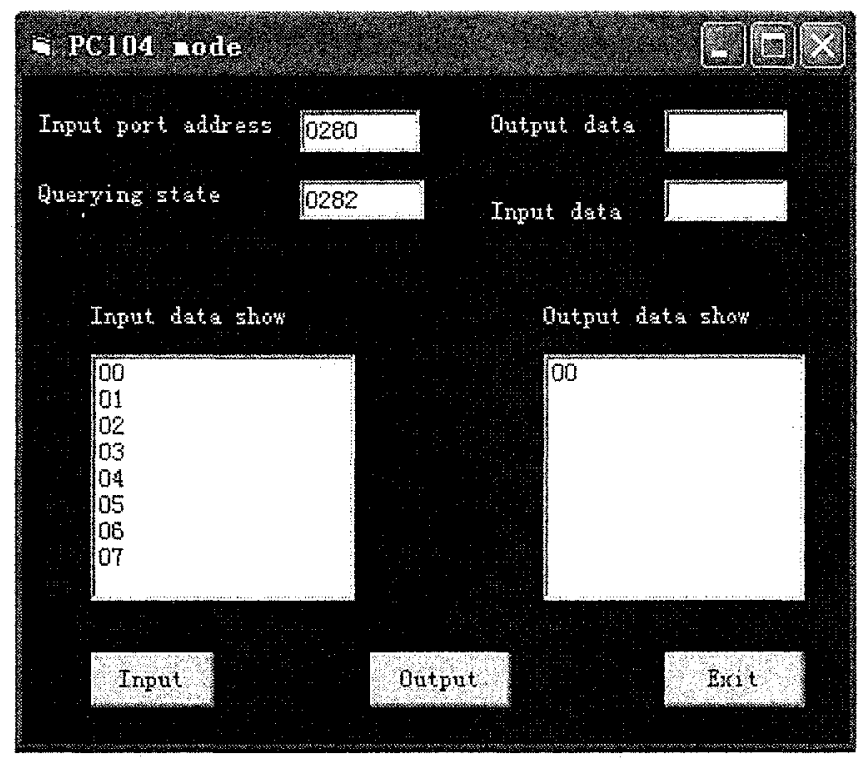


Figure3. Interface of node-to-node test

The test result proved that the adapter could perform the communication between CAN Bus and PC104 system accurately and rapidly. The test result was satisfactory

\section{SUMMARY}

An intelligent adapter between CAN bus network and PC/104 system was developed. It has following characteristics: (a) The adapter did not impropriate main board MAB. It only needed four free port-settings so that it would not appear the problem of the resource disturbance. (b) Since the adapter used parallel communication mode the data transmission speed was very high. (c) The MCU of the adapter made it intelligent to transmit and receive CAN-data packets. It not only could minimize the adapter's size and power consumption, but also could reduce the tasks of the PC104, which was only needed to take charge of transmitting and receiving CAN-data packet without conducting maintenance works

\section{ACKNOWLEDGEMENTS}

This study was supported by National High Technology Research and Development Program of China (863 Program): Research and Demonstration for Digital Agriculture (2003AA209040).

\section{REFERENCES}

[1] Wu bin, Yang xueyou, Chang jie. The Research of Auto car-Body Visual Inspection Station Based on CAN. Computer Automated Measurement\& Control.1999, 7(3): $16 \sim 17$

[2] Scarlet A. Integrated control of agricultural tractors and implements: a review of potential opportunities relating to cultivation and crop establishment machinery. Computers and Electronics in Agriculture, 2001, 30: 167-191

[3] .Hu gungbin, Hu yunan. A Temperatures-Measuring System with Multi-drop Capability Based on CAN. Automation \& Instrumentation.1999, 14(5) : 27 29

[4] Yang gongxun, Zhou chunliang, Yang yancong. Design of an Embedded DC Control and Monitoring Device Based on the PC104 Industry Controller. Industry and Mine Automation.2002 (2) : $25 \sim 27$ 
[5] .Liuhui. Research and Development on a Field Computer used in Farm Machinery. [Master's thesis], Beijing: China Agriculture University, 2003

[6] Wu chunxue, Hong kui, Lei Ming. The Vehicular Automatic Van Blender System Based on PC104 Computer. Computer Development \& Applications, 2003,16(5) : $21 \sim 22$

[7] Liu wei. The application of CAN controller SJA1000. Nanjing engineering institute.2002, 2(1): $32 \sim 35$

[8] .Philips semiconductors SJA1000 stand-along CAN controller. DATA SHEET.1997 